\title{
Shortcomings of the Multiple Tube Test for Bacteria in Drinking Water
}

\author{
Sayan Bhattacharyya
}

Department of Microbiology, All India Institute of Hygiene and Public Health, Kolkata, India

\section{ABSTRACT}

Background: The multiple tube test is used to Assess total and faecal coliforms in drinking water. It depends on colour change and gas formation but often identifies the bacteria wrongly. Many a times bacteria are missed or important bacteria are not identified by this test.

Methods: Here we propose another new method combining subculture and biochemical tests to complement this test so that identification is better.

Result: The new method was found to be very good and can supplement Eijkman test and Multiple tube test for better identification of coliforms and other bacteria from drinking water.

Conclusion: The new tests are simple and can be safely used in place of cumbersome conventional tests and other molecular tests for identification of bacteria in water.

Keywords: Drinking Water, Coliforms, Multiple Tube Test

\section{Introduction}

Multiple tube test (MTT), also called presumptive coliform test, is used to assess the quality of drinking water. In this test, a series of tubes containing media like single and double strength MacConkey broth is used to inoculate drinking water and culture $\mathrm{it}^{\left[{ }^{11}\right.}$. After usually 48 hours of incubation time at a given temperature, each tube showing colour change and gas formation is regarded as "presumptive positive" ; the gas indicates the possible presence of total coliforms. This test, also called the multiple tube fermentation test, also needs an incubation time of 96 hours $^{[2]}$. This test is over a 100 years old now and positive tubes have to be reconfirmed for fecal coliforms by Eijkman test using Tryptone water and brilliant green lactose bile broth. Fecal coliforms, if present, produce gas in the brilliant green broth and produce indole from tryptone water, when subcultured from MacConkey broth and incubated for 24 hours at 44 degree $\mathrm{C}$ or 46 degree $\mathrm{C}$ in the original method of Eijkman ${ }^{[3]}$. These tests are hence time consuming. So we proposed a new method, or subculture of different dilutions of MacConkey broth of the multiple tube test on to Nutrient broth to detect colonies specifically.

\section{Materials and Methods}

From January to June 2018, all drinking water specimens received in the laboratory of the institute, were processed by original multiple tube method(MTT) and Eijkman test using Brilliant green bile broth and Tryptone water, and also by our method. In this method, 10 microlitre of inoculum from all the turbid tubes of the MTT from different dilutions, were subcultured on Nutrient agar plates (Himedia, India) and were incubated at 37 degree C aerobically overnight. Next day, colonies appeared and were processed by Gram stain, Oxidase and other biochemical and phenotypic tests like Indole production, motility, citrate utilisation, urease activity, acid and $\mathrm{H}_{2} \mathrm{~S}$ production in TSI(Triple Sugar Iron) slant etc. Positive findings using both the methods were noted.

\section{Results}

Frequently it was found that bacterial isolates, especially Pseudomonas aeruginosa were missed by MTT and Eijkman tests but picked up by the new method. Also indole negative isolates of $E$. coli were missed and came out negative by the Eijkman test. Also the new method combining subculture and biochemicals helped in picking up isolates of Enterococcus casseliflavus (from one sample) and non fermenting Gram negative bacilli like Stenotrophomonas maltophilia, which were not done by MTT and Eijkman test. Isolates like Aeromonas spp. were also identified by this new method. Aeromonas asp. Were confirmed by salt tolerance test, Ampicillin resistance using disk diffusion test and string test. They were completely missed by the conventional method. Also it was found by the subculture-based new method that in different dilutions from same sample, different bacteria were recovered. These were not seen or appreciated in 
MTT method. Many a times multiple strains or isolates were recovered by this new plating technique, as compared to MTT where this was impossible. Cost incurred was also same in both tests. However time required is 24 hours more in this new method, but the specificity of identification is better.

\section{Discussion}

Bacteria like non fermenting gram negative rods are important to cause water borne infections and should be detected ${ }^{[4]}$. Similar is the case with Aeromonas spp. which can cause waterborne infections like gastroenteritis and is notoriously resistant to Chlorine disinfectants ${ }^{[5]}$. Aeromonas spp. in drinkig water should be of teremendous public health concern because of their ability to form toxins and biofilms ${ }^{[5]}$. Reports mention use of specific tests like Multiple tube test using alkaline peptone water and then subculture on to Ampicillin blood agar for detecting Aeromonas $\mathrm{spp}^{[5]}$. Even Pseudomonas aeruginosa is harmful if detected in drinking water because in people taking antibiotics to which it is resistant, this pathogen can cause gastritis and also skin infections and aerosol mediated respiratory infections ${ }^{[6]}$. Coliforms are the universally accepted indicator bacteria for monitoring bacterial water quality because they are found in human stool and are usually resistant to chlorination ${ }^{[7]}$. However the conventional tests are often inefficacious in picking out coliforms in water. Also Eijkman tests only identifies indole positive $E$. coli but misses the indole negative isolates of this pathogen. These are the shortcomings of the MTT and Eijkman test which can be overcome by the new method using subculture from turbid MTT tubes and further identification. It is more accurate and can be further strengthened by subculture on more selective and special media. As far as we know, nobody has tried this method for better identification of medically important and pathogenic bacteria from water samples. So MTT and Eijkman test are no longer only sufficient for generating a good and accurate report on bacteriological quality of drinking water from a lab. The new method of subculture-based identification can supplement the conventional methods.

\section{Conclusion}

Subculture based method is an adjunct to conventional methods for better reporting on water bacteriology.

\section{References:}

1. Annex 5 Multiple-tube method for thermotolerant (faecal) coliforms. http://www.who.int/water_sanitation health/ water-quality/small-community-management/2edvol3i.pdf. Last accessed 24th June 2018.

2. Hsieh JJ. A Comparison of the Multiple-Tube Fermentation Method and the Colitag Method for the Detection of Waterborne Coliform Bacteria. https://nature.berkeley.edu/ classes/es196/projects/2001final/Hsieh.pdf. Last accessed 24th June 2018.

3. Batty-Smith CG. The Eijkman test for faecal coli in the bacteriological examination of water supplies'. 1-44. https:// www.ncbi.nlm.nih.gov/pmc/articles/PMC2199795/pdf/ jhyg00198-0064.pdf. Last accessed 24th June 2018.

4. Zanetti F, de Luca G, Leoni E, Sachetti R. Occurrence of non-fermenting gram negative bacteria in drinking water dispensed from point-of-use microfiltration devices. Ann Agric Environ Med. 2014;21: 29-34

5. Razzolini MTP, Guinther WMR, Martone-Rocha S, de Luca $\mathrm{HD}$, Cardoso MRA. Aeromonas presence in drinking water from collective reservoirs and wells in periurban area in brazil. Brazilian J Microbiol 2010;41: 694-699.

6. Mena KD, Gerba CP. Risk assessment of Pseudomonas aeruginosa in water. Rev Environ Contam Toxicol. 2009;201:71-115.

7. Chapter 2 - water quality monitoring, standards and treatment. http://www.fao.org/docrep/x5624e/x5624e05. $\mathrm{htm} \# 2.2 .3$ bacteriological tests. Last accessed 6th August 2018 .

*Corresponding author:

Dr Sayan Bhattacharyya, Assistant Professor, Department of Microbiology, All India Institute of Hygiene and Public Health, Kolkata-106. India Phone: +91 9432488669, 9006621729

Email: sayan.bhattacharyya@yahoo.com

Financial or other Competing Interests: None. 